\title{
Lognormal Moment Closures for Biochemical Reactions
}

\author{
Abhyudai Singh and João Pedro Hespanha
}

\begin{abstract}
In the stochastic formulation of chemical reactions, the dynamics of the the first $\mathscr{M}$-order moments of the species populations generally do not form a closed system of differential equations, in the sense that the time-derivatives of first $\mathscr{M}$-order moments generally depend on moments of order higher than $\mathscr{M}$. However, for analysis purposes, these dynamics are often made to be closed by approximating the needed derivatives of the first $\mathscr{M}$-order moments by nonlinear functions of the same moments. These functions are called the moment closure functions.
\end{abstract}

Recent results have introduced the technique of derivativematching, where the moment closure functions are obtained by first assuming that they exhibit a certain separable form, and then matching time derivatives of the exact (not closed) moment equations with that of the approximate (closed) equations for some initial time and set of initial conditions. However, for multi-species reactions these results have been restricted to second order truncations, i.e, $\mathscr{M}=2$.

This paper extends these results by providing explicit formulas to construct moment closure functions for any arbitrary order of truncation $\mathscr{M}$. We show that with increasing $\mathscr{M}$ the closed moment equations provide more accurate approximations to the exact moment equations. Striking features of these moment closure functions are that they are independent of the reaction parameters (reaction rates and stoichiometry) and moreover the dependence of higher-order moment on lower order ones is consistent with the population being jointly lognormally distributed. To illustrate the applicability of our results we consider a simple bi-molecular reaction. Moment estimates from a third order truncation are compared with estimates obtained from a large number of Monte Carlo simulations.

\section{INTRODUCTION}

The time evolution of a spatially homogeneous mixture of chemically reacting molecules is often modeled using a stochastic formulation, which takes into account the inherent randomness of thermal molecular motion. This formulation is superior to the traditional deterministic formulation of chemical kinetics and is motivated by complex reactions inside living cells, where small populations of key reactants can set the stage for significant stochastic effects [1]-[2].

In the stochastic formulation, the time evolution of the system is described by a single equation for a grand probability function, where time and species populations

This material is based upon work supported by the Institute for Collaborative Biotechnologies through grant DAAD19-03-D-0004 from the U.S. Army Research Office and by the National Science Foundation under Grant No. CCR-0311084.

A.Singh and J.P.Hespanha are with the Center for Control Engineering and Computation University of California, Santa Barbara, CA 93101. abhidengineering.ucsb.edu, hespanha@ece.ucsb.edu appear as independent variables, called the Master equation [3]. However, this equation can only be solved for relatively few, highly idealized cases and generally Monte Carlo simulation techniques are used [4]-[5]. Since one is often interested in only the first and second order moments for the number of molecules of the different species involved, much effort can be saved by applying approximate methods to produce these low-order moments, without actually having to solve for the probability density function. Various such approximate methods have been developed, for example, using the Fokker-Plank approximation or expanding the Master equation [3].

In this paper, an alternative method for estimating low-order statistical moments is introduced for a general class of elementary chemical reactions involving $n$ chemical species and $K$ reactions. To derive differential equations for the time evolution of moments of the populations, the set of chemical reactions is first modeled as a Stochastic Hybrid System (SHS) with state $\mathbf{x}=\left[\mathbf{x}_{1}, \ldots, \mathbf{x}_{n}\right]^{T}$, where $\mathbf{x}_{j}$ denotes the population of specie $X_{j}$ involved in the chemical reaction. In order to fit the framework of our problem, this SHS has trivial continuous dynamics $\dot{\mathbf{x}}=0$, with reset maps and transitional intensities defined by the stoichiometry and the reaction rates of the reactions, respectively. In essence, if no reaction takes place, the population of species remain constant and whenever a reaction takes place, the corresponding reset map is "activated" and the population is reset according to the stoichiometry of the reaction. Details for the stochastic modeling of chemical reactions are presented in Sec. II. The time evolution of the moments is then obtained using results from the SHS literature [6].

We show in Section III, that the time evolution of a moment of $\mathbf{x}$ of order $m^{*}$ is given as a linear combination of moments of order up to $m^{*}+1$. Hence, if one creates an infinite vector $\mu_{\infty}$ containing all the statistical moments of $\mathbf{x}$, the dynamics of this vector is governed by an infinite-dimensional linear ordinary differential equation

$$
\dot{\mu}_{\infty}=A_{\infty} \mu_{\infty}
$$

As the infinite-dimensional moment dynamics cannot be solved analytically, we perform an $\mathscr{M}^{\text {th }}$ order trunction, i.e., we focus our attention on a vector $\mu$ containing all moments of $\mathbf{x}$ up to order $\mathscr{M}$. Its dynamics, given by

$$
\dot{\mu}=A \mu+B \bar{\mu}
$$

for some matrices $A$ and $B$, is not closed, in the sense that the time derivative of the vector $\mu$ depends on $\bar{\mu}$ which is a 
vector containing moments of $\mathbf{x}$ of order $\mathscr{M}+1$. For analysis purposes, we close the above system by approximating each element of $\bar{\mu}$ as a nonlinear function $\varphi(\mu)$ of moments up to order $\mathscr{M}$. This procedure is commonly referred to as moment closure. We call $\varphi(\mu)$ the moment closure function for the corresponding element of $\bar{\mu}$. We denote the state of the new system, which is an approximation for $\mu$ as $v$, with dynamics given by

$$
\dot{v}=A v+B \bar{\varphi}(v)
$$

where $\bar{\varphi}$ is a vector of moment closure functions $\varphi$ and is referred to as the truncated moment dynamics.

In [7], a general procedures to construct these moment closure functions was outlined. This procedure was based on first assuming a certain separable form for each element $\varphi$ of $\bar{\varphi}$ and then matching time derivatives of $\mu$ and $v$ in (2) and (3) respectively, at some initial time $t_{0}$, for every deterministic initial condition of the form $\mathbf{x}\left(t_{0}\right)=\overline{\mathbf{x}}$ with probability one. The choice of initial conditions is justified by the fact that the class of deterministic distributions forms a natural basis for the infinite dimensional space $\Omega_{\infty}$ containing every possible state $\mu_{\infty}$ of (1). For a second order of truncation $(\mathscr{M}=2)$, [8] provided explicit formulas to construct these moment closure functions.

Although second order truncations provide good estimates, it is typically beneficial to consider higher order truncations because they lead to better approximations and often reduce the errors by orders of magnitude. This paper builds upon the result of [8] and provides explicit formulas to construct moment closure functions for any arbitrary order of truncation $\mathscr{M}$. In particular, we provide in Section IV moment closure functions that exhibit the property that when $\mu\left(t_{0}\right)=v\left(t_{0}\right)$, we have

$$
\frac{d^{i} \mu\left(t_{0}\right)}{d t^{i}}=\frac{d^{i} v\left(t_{0}\right)}{d t^{i}}+\varepsilon_{i}(\overline{\mathbf{x}}), \quad \forall i \geq 1
$$

for every deterministic initial conditions of the form $\mathbf{x}\left(t_{0}\right)=\overline{\mathbf{x}}$ with probability one for every integer $\overline{\mathbf{x}}$. In the equation above, each entry of the vector $\frac{d^{i} \mu\left(t_{0}\right)}{d t^{i}}$ is a polynomial in $\overline{\mathbf{x}}$, whose degree exceeds by at least $\mathscr{M}$ the degree of the corresponding entry of the error vector $\varepsilon_{i}(\overline{\mathbf{x}})$. Thus, with increasing $\mathscr{M}$, the truncated moment dynamics $v(t)$ provides a more accurate approximation to the moments in $\mu(t)$. The striking feature of the moment closure constructed in Section IV is that they are independent of the reaction parameters (reaction rates and stoichiometry) and moreover the dependence of higher-order moment on lower order ones is consistent with $\mathbf{x}$ being jointly lognormally distributed, in spite of the fact that the procedure used to construct $\varphi$ did not make any assumption on the distribution of the population

To illustrate the applicability of our results, in Section V we consider a simple bi-molecular chemical reaction. Moment estimates obtained from the truncated moment dynamics are
TABLE I

$h_{i}(\mathbf{x})$ AND $c_{i}$ FOR DIFFERENT REACTION TYPES.

\begin{tabular}{lcc}
\hline Reaction $\mathrm{R}_{\mathrm{i}}$ & $h_{i}(\mathbf{x})$ & $c_{i}$ \\
\hline$X_{j} \longrightarrow *$ & $\mathbf{x}_{j}$ & $k_{i}$ \\
$X_{j}+X_{t} \longrightarrow *, \quad(t \neq j)$ & $\mathbf{x}_{j} \mathbf{x}_{t}$ & $\frac{k_{i}}{V}$ \\
$2 X_{j} \longrightarrow *$ & $\frac{1}{2} \mathbf{x}_{j}\left(\mathbf{x}_{j}-1\right)$ & $\frac{2 k_{i}}{V}$ \\
\hline
\end{tabular}

compared with estimates obtained from a large number of Monte Carlo simulations.

\section{Stochastic Modeling of Chemically Reacting SYSTEMS}

Consider a system of $n$ species $X_{j}, j \in\{1, \ldots, n\}$ inside a fixed volume $V$ involved in $K$ reactions of the form

$$
R_{i}: u_{i 1} X_{1}+\ldots+u_{i n} X_{n} \stackrel{c_{i}}{\longrightarrow} v_{i 1} X_{1}+\ldots+v_{i n} X_{n}+*
$$

for all $i \in\{1, \ldots, K\}$, where $u_{i j} \in \mathbb{N}_{\geq 0}$ is the stoichiometry associated with the $j^{t h}$ reactant in the $i^{t h}$ reaction, $v_{i j} \in \mathbb{N}_{\geq 0}$ is the stoichiometry associated with the $j^{t h}$ product in the $i^{t h}$ reaction, and the symbol $*$ represent products other than the species $X_{j}$. As all chemical reactions occur in a series of elementary reactions [9], which are generally uni- or bimolecular, we assume that

$$
u_{i 1}+\ldots+u_{\text {in }} \leq 2, \quad \forall i \in\{1, \ldots, K\}
$$

and hence, we only allow reactions which have the form given in the first column of Table I. The reaction parameter $c_{i}$ characterizes the reaction $R_{i}$ and, together with the stoichiometry, defines the probability that a particular reaction takes place in an "infinitesimal" time interval $(t, t+d t]$. This probability is given by the product $c_{i} h_{i} d t$ where $h_{i}$ is the number of distinct molecular reactant combinations present in $V$ at time $t$ for the reaction $R_{i}$. Table I shows the value of $h_{i}$ for different reaction types [4]. In this table and in the sequel, we denote by $\mathbf{x}_{j}$, the number of molecules of the species $X_{j}$ in the volume $V$ and $\mathbf{x}:=\left[\mathbf{x}_{1}, \ldots, \mathbf{x}_{n}\right]^{T} \in \mathbb{R}^{n}$. The reaction parameter $c_{i}$ is related to the reaction rate $k_{i}$ in the deterministic formulation of chemical kinetics by the formulas shown in the right-most column of Table I.

To model the time evolution of the number of molecules $\mathbf{x}_{1}, \mathbf{x}_{2}, \ldots, \mathbf{x}_{n}$, a special class of Stochastic Hybrid Systems (SHS) were introduced in [7] . More specifically, to fit the framework of our problem, these systems are characterized by trivial dynamics

$$
\dot{\mathbf{x}}=0, \quad \mathbf{x}=\left[\mathbf{x}_{1}, \ldots, \mathbf{x}_{n}\right]^{T},
$$

a family of $K$ reset maps

$$
\mathbf{x}=\phi_{i}\left(\mathbf{x}^{-}\right), \quad \phi_{i}: \mathbb{R}^{n} \rightarrow \mathbb{R}^{n},
$$

and a corresponding family of $K$ transition intensities

$$
\lambda_{i}(\mathbf{x}), \quad \lambda_{i}: \mathbb{R}^{n} \rightarrow[0, \infty)
$$


for every $i \in\{1, \ldots, K\}$. Each of the reset maps $\phi_{i}(\mathbf{x})$, and corresponding transition intensities $\lambda_{i}(\mathbf{x})$ are uniquely defined by the $i^{t h}$ reaction and are given by

$$
\mathbf{x} \mapsto \phi_{i}(\mathbf{x})=\left[\begin{array}{c}
\mathbf{x}_{1}-u_{i 1}+v_{i 1} \\
\mathbf{x}_{2}-u_{i 2}+v_{i 2} \\
\vdots \\
\mathbf{x}_{n}-u_{i n}+v_{i n}
\end{array}\right], \lambda_{i}(\mathbf{x})=c_{i} h_{i}(\mathbf{x})
$$

for every $i \in\{1, \ldots, K\}$. In essence, if no reaction takes place, the state remains constant and whenever the $i^{\text {th }}$ reaction takes place, $\phi_{i}(\mathbf{x})$ is "activated" and the state $\mathbf{x}$ is reset according to (9). Furthermore, the probability of an activation taking place in an "infinitesimal" time interval $(t, t+d t]$ is given by $\lambda_{i}(\mathbf{x}) d t$.

\section{Moment Dynamics}

Given a vector $\mathbf{m}=\left(m_{1}, m_{2}, \ldots, m_{n}\right) \in \mathbb{N}_{\geq 0}^{n}$ of $n$ greater than equal to zero integers, we define the (uncentered) moment of $\mathbf{x}$ associated with $\mathbf{m}$ to be

$$
\mu^{(\mathbf{m})}(t)=\mathbf{E}\left[\mathbf{x}^{(\mathbf{m})}(t)\right], \quad \forall t \geq 0
$$

where $\mathbf{E}$ stands for the expected value and

$$
\mathbf{x}^{(\mathbf{m})}:=\mathbf{x}_{1}^{m_{1}} \mathbf{x}_{2}^{m_{2}} \cdots \mathbf{x}_{n}^{m_{n}}
$$

The sum $\sum_{j=1}^{n} m_{j}$ is called the order of the moment $\mathbf{m}$. The time evolution of moments is given by the following result, which is a straightforward application of Theorem 1 in [6] to the SHS (6)-(8).

Theorem 1: For every differentiable function $\psi: \mathbb{R}^{n} \rightarrow \mathbb{R}$ we have that

$$
\frac{d \mathbf{E}[\psi(\mathbf{x})]}{d t}=\mathbf{E}[(\mathbf{L} \psi)(\mathbf{x})]
$$

where, $\forall \mathbf{x} \in \mathbb{R}^{n}$,

$$
(\mathbf{L} \psi)(\mathbf{x})=\sum_{i=1}^{K}\left(\psi\left(\phi_{i}(\mathbf{x})\right)-\psi(\mathbf{x})\right) \lambda_{i}(\mathbf{x})
$$

We now use the above theorem to show that the time derivative of a moment $\mu^{(\mathbf{m})}$ of order $m^{*}$ is given by a linear combination of moments of orders upto $m^{*}+1$. Using (9) and replacing $\psi(\mathbf{x})$ with $\mathbf{x}^{(\mathbf{m})}$ in (12) and (13), we have that

$$
\dot{\mu}^{(\mathbf{m})}=\mathbf{E}[(\mathbf{L} \psi)(\mathbf{x})]
$$

where $^{1}$

$$
\begin{aligned}
(\mathbf{L} \psi)(\mathbf{x}) & =\sum_{i=1}^{K} c_{i} h_{i}(\mathbf{x})\left\{\phi_{i}(\mathbf{x})^{(\mathbf{m})}-\mathbf{x}^{(\mathbf{m})}\right\} \\
& =\sum_{i=1}^{K} c_{i} h_{i}(\mathbf{x})\left\{\left[\prod_{j=1}^{n}\left(\mathbf{x}_{j}-u_{i j}+v_{i j}\right)^{m_{j}}\right]-\mathbf{x}^{(\mathbf{m})}\right\} \\
& =\sum_{i=1}^{K} c_{i} h_{i}(\mathbf{x})\left\{\left[\prod_{j=1}^{n} \sum_{t=0}^{m_{j}} \mathrm{C}_{t}^{m_{j}} \mathbf{x}_{j}^{m_{j}-t} a_{i j}^{t}\right]-\mathbf{x}^{(\mathbf{m})}\right\} \\
& =\sum_{i=1}^{K} c_{i} h_{i}(\mathbf{x})\left\{\sum_{j=1}^{n} \mathrm{C}_{1}^{m_{j}} \frac{\mathbf{x}^{(\mathbf{m})}}{\mathbf{x}_{j}} a_{i j}+\ldots\right\}
\end{aligned}
$$

and $a_{i j}:=v_{i j}-\mu_{i j}$. Since $\mathbf{x}^{(\mathbf{m})}$ is a monomial of degree $m^{*}$, and $h_{i}(\mathbf{x})$ is a polynomial of degree no larger than 2 , the polynomial

$$
\sum_{i=1}^{K} \sum_{j=1}^{n} c_{i} h_{i}(\mathbf{x}) \mathrm{C}_{1}^{m_{j}} a_{i j} \frac{\mathbf{x}^{(\mathbf{m})}}{\mathbf{x}_{j}}
$$

will contain monomials of degree up to $m^{*}+1$, which will contribute to moments of order $m^{*}+1$ in $\dot{\mu}^{(\mathbf{m})}$ given by (14). Lesser order moments will appear from the other terms in the binomial expansion. Hence, if one stacks all moments in an infinite vector

$$
\mu_{\infty}=\left[\mu^{(\mathbf{m} 1)}, \mu^{(\mathbf{m} 2)}, \cdots\right]^{T}, \mathbf{m} p \in \mathbb{N}_{\geq 0}^{n}, \forall p \in\{1,2, \ldots\}
$$

its dynamics can be written as

$$
\dot{\mu}_{\infty}=A_{\infty} \mu_{\infty}
$$

for some infinite matrix $A_{\infty}$. As the above infinite dimensional system cannot be solved analytically, we truncate (16) by creating a vector

$$
\mu=\left[\mu^{(\mathbf{m} 1)}, \mu^{(\mathbf{m} 2)}, \ldots, \mu^{(\mathbf{m} k)}\right]^{T} \in \mathbb{R}^{k}
$$

containing the top $k$ elements of $\mu_{\infty}$ which correspond to the lower-order moments of interest. Then, (16) can be re-written as

$$
\dot{\mu}=I_{k \times \infty} A_{\infty} \mu_{\infty}=A \mu+B \bar{\mu}
$$

where $\mu \in \mathbb{R}^{k}, I_{k \times \infty}$ denotes a matrix composed of the first $k$ rows of the infinite identity matrix and $\bar{\mu} \in \mathbb{R}^{r}$ contains all the moments that appear in the first $k$ elements of $A_{\infty} \mu_{\infty}$ but that do not appear in $\mu$.

In this paper we let the vector $\mu \in \mathbb{R}^{k}$ contain all the moments of $\mathbf{x}$, of order upto $\mathscr{M} \in \mathbb{N}_{\geq 2}$, i.e., we consider an $\mathscr{M}^{\text {th }}$ order truncation. With this, the evolution of vector $\mu$ can be written as (18) for suitably defined matrices $A$ and $B$ obtained from (14) with $\bar{\mu} \in \mathbb{R}^{r}$ being a vector of moments of order $\mathscr{M}+1$. Our goal now is to approximate (18) by a finite-dimensional nonlinear ODE of the form

$$
\begin{gathered}
\dot{v}=A v+B \bar{\varphi}(v), v=\left[v^{(\mathbf{m} 1)}, v^{(\mathbf{m} 2)}, \ldots, v^{(\mathbf{m} k)}\right]^{T} \\
{ }^{1} \mathrm{C}_{h}^{\ell} \text { is defined as follows: } \forall \ell, h \in \mathbb{N}_{\geq 0} \\
\mathrm{C}_{h}^{\ell}= \begin{cases}\frac{\ell !}{(\ell-h) ! h !}, & \ell \geq h \\
0, & \ell<h\end{cases}
\end{gathered}
$$

where $\ell$ ! denotes the factorial of $\ell$. 
where the map $\bar{\varphi}: \mathbb{R}^{k} \rightarrow \mathbb{R}^{r}$ should be chosen so as to keep $v(t)$ close to $\mu(t)$. This procedure is commonly referred to as moment closure. We call (19) the truncated moment dynamics and each element $\varphi^{(\overline{\mathbf{m}})}(\mu)$ of $\bar{\varphi}(\mu)$ the moment closure function for the corresponding element $\mu^{(\overline{\mathbf{m}})}$ in $\bar{\mu}$.

When a sufficiently large but finite number of derivatives of $\mu(t)$ and $v(t)$ match point-wise, then, the difference between solutions to (18) and (19) remains close on a given compact time interval. This follows from a Taylor series approximation argument. To be more precise, for each $\delta>0$ and $T \in \mathbb{R}$, there exists an integer $N$, sufficiently large, for which the following result holds: Assuming that for every $t_{0} \geq 0$,

$$
\mu\left(t_{0}\right)=v\left(t_{0}\right) \Rightarrow \frac{d^{i} \mu\left(t_{0}\right)}{d t^{i}}=\frac{d^{i} v\left(t_{0}\right)}{d t^{i}}, \forall i \in\{1,2, \ldots, \mathrm{N}\}
$$

where $\frac{d^{i} \mu\left(t_{0}\right)}{d t^{i}}$ and $\frac{d^{i} v\left(t_{0}\right)}{d t^{i}}$ represent the $i^{t h}$ time derivative of $\mu\left(t_{0}\right)$ and $v\left(t_{0}\right)$ along the trajectories of system (16) and (19) respectively. Then,

$$
\|\mu(t)-v(t)\| \leq \delta, \forall t \in\left[t_{0}, T\right],
$$

along solutions to (16) and (19), where $\mu$ denotes a vector containing the first $k$ elements of $\mu_{\infty}$.

\section{Construction of Approximate Truncations}

In this section we construct approximate truncated moment dynamics for the general set of reactions introduced in (4). Our goal is to approximate (18) by a finite-dimensional nonlinear ODE of the form (19) with $\bar{\varphi}: \mathbb{R}^{k} \rightarrow \mathbb{R}^{r}$ chosen so that the condition (20) holds. After replacing (18) and (19) in (20), we obtain a PDE on $\bar{\varphi}$. We seek solutions $\bar{\varphi}$ to this PDE for which each entry $\varphi^{(\overline{\mathbf{m}})}$ has the following separable form

$$
\varphi^{(\overline{\mathbf{m}})}(v)=\prod_{p=1}^{k}\left(v^{(\mathbf{m} p)}\right)^{\gamma_{p}}=v^{(\gamma)}, \quad \gamma=\left(\gamma_{1}, \ldots, \gamma_{k}\right)
$$

where $\gamma_{p}$ are some appropriately chosen constants.

\section{A. Derivative matching for a basis}

In general, it will not be possible to find constants $\gamma_{p}$ such that (20) holds for every initial vector $\mu_{\infty}\left(t_{0}\right)$ in the set $\Omega_{\infty}$, containing all possible infinite vectors with the moments of $\mathbf{x}$. We therefore restrict $\mu_{\infty}\left(t_{0}\right)$ to belong to a set $\mathscr{B} \subset \Omega_{\infty}$, containing only moments consistent with deterministic initial conditions. The rationale for this choice lies in the fact that the set $\mathscr{B}$ forms a natural basis for the infinite dimensional space $\Omega_{\infty}$ containing any vector $\mu_{\infty}$ [10].

Often it is still not possible to find $\gamma_{p}$ for which (20) holds exactly for $\mu_{\infty}\left(t_{0}\right) \in \mathscr{B}$. We will therefore relax this condition and simply demand the following

$$
\mu\left(t_{0}\right)=v\left(t_{0}\right) \Rightarrow \frac{d^{i} \mu\left(t_{0}\right)}{d t^{i}}=\frac{d^{i} v\left(t_{0}\right)}{d t^{i}}+\mathbf{E}\left[\varepsilon_{i}\left(\mathbf{x}\left(t_{0}\right)\right)\right],
$$

$\forall i \in\{1,2, \ldots, N\}$, where $\varepsilon_{i}: \mathbb{R}^{n} \rightarrow \mathbb{R}^{k}$ is a vector-valued polynomial function on $\mathbf{x}\left(t_{0}\right)$. One can think of (23) as an approximation to (20) which will be valid as long as the derivatives in $\frac{d^{i} \mu\left(t_{0}\right)}{d t^{i}}$ dominate the errors in $\mathbf{E}\left[\varepsilon_{i}\left(\mathbf{x}\left(t_{0}\right)\right)\right]$.

Before stating our result, the proof of which is omitted due to space considerations, we define for vectors $\hat{\mathbf{m}}=\left(\hat{m}_{1}, \ldots, \hat{m}_{n}\right)$ and $\check{\mathbf{m}}=\left(\check{m}_{1}, \ldots, \check{m}_{n}\right)$

$$
\mathrm{C}_{(\check{\mathbf{m}})}^{(\hat{\mathbf{m}})}:=\mathrm{C}_{\check{m}_{1}}^{\hat{m}_{1}} \mathrm{C}_{\check{m}_{2}}^{\hat{m}_{2}} \ldots \mathrm{C}_{\check{m}_{n}}^{\hat{m}_{n}}
$$

Also we let $\bar{n}=k-n^{*}+1$, where $n^{*}$ denotes the row in the vector $\mu$ from where the $\mathscr{M}^{\text {th }}$ order moments of $\mathbf{x}$ starts appearing.

Theorem 2: Assume that for each element $\mu^{(\overline{\mathbf{m}})}$ of $\bar{\mu}$, the corresponding moment closure function $\varphi^{(\overline{\mathbf{m}})}$ in $\bar{\varphi}$ is chosen according to (22) with $\gamma=\left(\gamma_{1}, \ldots, \gamma_{k}\right)$ taken as the unique solution of the following system of linear equations

$$
\mathbf{C}_{(\mathbf{m} s)}^{(\overline{\mathbf{m}})}=\sum_{p=1}^{k} \gamma_{p} \mathbf{C}_{(\mathbf{m} s)}^{(\mathbf{m} p)}, \quad \forall s=\{1, \ldots, k\} .
$$

Then, with initial conditions $\mu_{\infty}\left(t_{0}\right) \in \mathscr{B}$ and $\mathbf{x}\left(t_{0}\right)=\overline{\mathbf{x}}=$ $\left[\bar{x}_{1}, \ldots, \bar{x}_{n}\right]^{T}$ with probability one, we have that

$$
\begin{aligned}
\mu\left(t_{0}\right)=v\left(t_{0}\right) & \Rightarrow \frac{d \mu\left(t_{0}\right)}{d t}=\frac{d v\left(t_{0}\right)}{d t} \\
& \Rightarrow \frac{d^{2} \mu\left(t_{0}\right)}{d t}=\frac{d^{2} v\left(t_{0}\right)}{d t}+\varepsilon_{2}(\overline{\mathbf{x}})
\end{aligned}
$$

where the last $\bar{n}$ elements of $\varepsilon_{2}(\overline{\mathbf{x}})$ are polynomials in $\overline{\mathbf{x}}$ of degree 2 and all other elements are zero.

In the above Theorem, the degrees of the bottom $\bar{n}$ polynomial entries of the vector $\frac{d^{2} \mu\left(t_{0}\right)}{d t}$ is larger than the degree of the corresponding polynomial elements of the error vector $\varepsilon_{2}(\overline{\mathbf{x}})$ by $\mathscr{M}$. Thus, for a second order of truncation $\mathscr{M}=2$ and 10 molecules for all species, the elements of $\frac{d^{2} \mu\left(t_{0}\right)}{d t}$ are typically two orders of magnitude larger than $\varepsilon_{2}(\overline{\mathbf{x}})$.

It can be verified that with $\gamma$ chosen as in Theorem 2 , the moment closure functions so obtained also match derivatives of order higher than 2 in (27) with small errors. Using symbolic manipulation in Mathematica, for $n \in\{1,2,3\}$ and $i \in\{3,4,5\}$ we have that the degree of the polynomial entries of $\frac{d^{i} \mu\left(t_{0}\right)}{d t^{i}}$ are larger then the degree of the corresponding polynomial entries in the error vector $\varepsilon_{i}(\overline{\mathbf{x}})=\frac{d^{i} \mu\left(t_{0}\right)}{d t^{i}}-\frac{d^{i} v\left(t_{0}\right)}{d t^{i}}$ by $\mathscr{M}$. We conjecture that this is true $\forall n \in \mathbb{N}$ and $\forall i \in \mathbb{N}$ but we only verified it for $n$ up to 3 and $i$ up to 5 . Hence, with increasing $\mathscr{M}$, the truncated moment dynamics $v(t)$ should provide a more accurate approximations to the lower order moments $\mu(t)$.

\section{B. Moment closure functions}

In this section, we use (25) to construct moment closure functions for different higher order moments $\mu^{(\overline{\mathbf{m}})}$ 
corresponding to two-specie reactions $(n=2)$ and various orders of truncation $\mathscr{M}$. The reader is refereed to [11] and [10] for a discussion on moment closures for single specie chemical reactions.

We first consider a second order truncation $\mathscr{M}=2$, and hence,

$$
\mu=\left[\mu^{(1,0)}, \mu^{(0,1)}, \mu^{(2,0)}, \mu^{(0,2)}, \mu^{(1,1)}\right]^{T} .
$$

We recall that for $\mathscr{M}=2$, the vector $\bar{\mu}$ in (18) contains third order moments of $\mathbf{x}$. Using Theorem 2, the constants $\gamma_{p}$ are obtained for different third order moments. Table II summarizes the moment closure functions obtained for different third order moments. As one can see from this

TABLE II

MOMENT CLOSURE FUNCTION $\varphi^{(\overline{\mathbf{m}})}(\mu)$ FOR DIFFERENT THIRD ORDER MOMENTS $\mu^{(\overline{\mathbf{m}})}$ WITH $\mathscr{M}=2$ AND $n=2$.

\begin{tabular}{lc}
\hline$\mu^{\overline{\mathbf{m}}}$ & $\varphi^{(\overline{\mathbf{m}})}(\mu)$ \\
$\mu^{(3,0)}$ & $\left(\frac{\mu^{(2,0)}}{\mu^{(1,0)}}\right)^{3}$ \\
$\mu^{(0,3)}$ & $\left(\frac{\mu^{(0,2)}}{\mu^{(0,1)}}\right)^{3}$ \\
$\mu^{(2,1)}$ & $\left(\frac{\mu^{(2,0)}}{\mu^{(0,1)}}\right)\left(\frac{\mu^{(1,1)}}{\mu^{(1,0)}}\right)^{2}$ \\
$\mu^{(1,2)}$ & $\left(\frac{\mu^{(0,2)}}{\mu^{(1,0)}}\right)\left(\frac{\mu^{(1,1)}}{\mu^{(0,1)}}\right)^{2}$ \\
\hline
\end{tabular}

table, the moment closure function for $\mu^{(1,2)}$ can be directly obtained from that of $\mu^{(2,1)}$ by just switching the elements of vectors $\mathbf{m} p$ in $\varphi^{(2,1)}(\mu)$.

For a third order truncation $\mathscr{M}=3$ for which

$\mu=\left[\mu^{(1,0)}, \mu^{(0,1)}, \mu^{(2,0)}, \mu^{(0,2)}, \mu^{(1,1)}, \mu^{(3,0)}, \mu^{(0,3)}, \mu^{(2,1)}\right.$, $\left.\mu^{(1,2)}\right]^{T}$

Table III lists the different moment closure functions obtained for different fourth order moments of $\mathbf{x}$. We only list

TABLE III

MOMENT CLOSURE FUNCTION $\varphi^{(\overline{\mathbf{m}})}(\mu)$ FOR DIFFERENT FOURTH ORDER MOMENTS $\mu^{(\overline{\mathbf{m}})}$ WITH $\mathscr{M}=3$ AND $n=2$.

\begin{tabular}{cc}
\hline$\mu^{\overline{\mathbf{m}}}$ & $\varphi^{(\overline{\mathbf{m}})}(\mu)$ \\
\hline$\mu^{(4,0)}$ & $\frac{\mu^{(1,0)^{4}}}{\mu^{(2,0)^{2}}}\left(\frac{\mu^{(3,0)}}{\mu^{(2,0)}}\right)^{4}$ \\
$\mu^{(3,1)}$ & $\left(\frac{\mu^{(2,1)}}{\mu^{(1,1)}}\right)^{3}\left(\frac{\mu^{(1,0)}}{\mu^{(2,0)}}\right)^{3} \mu^{(3,0)} \mu^{(0,1)}$ \\
$\mu^{(2,2)}$ & $\frac{\left(\mu^{(2,1)} \mu^{(1,2)} \mu^{(0,1)} \mu^{(1,0)}\right)^{2}}{\left(\mu^{(1,1)}\right)^{4} \mu^{(2,0)} \mu^{(0,2)}}$ \\
\hline
\end{tabular}

a few of them, because the remaining moment closure functions for fourth order moments can be obtained from the ones above by switching elements as discussed above. One can see that the above moment closure functions are independent of the stoichiometry of the reactions, the reaction rates and the number of reactions. Another interesting observation is that the dependence of higher-order moment on lower order ones as given by the above moment closure functions is consistent with $\mathbf{x}$ being jointly lognormally distributed. The reader if referred to [10] for a more detailed discussion on this issue.

\section{EXAMPLE}

We now illustrate the use of moment closure techniques by developing moment estimates for the following bi-molecular reaction

$$
X_{1} \stackrel{c_{1}}{\longrightarrow} 2 X_{1}+X_{2}, \quad X_{1}+X_{2} \stackrel{c_{2}}{\longrightarrow} X_{2}
$$

The structure of this reaction is motivated by the nonlinear population gowth of aphid's (minute plant-feeding insects) [12]. Let $\mathbf{x}_{1}(t)$ and $\mathbf{x}_{2}(t)$ denote the populations of species $X_{1}$ and $X_{2}$. The time evolution of the populations $\mathbf{x}=\left[\mathbf{x}_{1}, \mathbf{x}_{2}\right]^{T}$ can be modeled by a SHS with continuous dynamics $\dot{\mathbf{x}}=0$ and two reset maps

$$
\begin{aligned}
& \mathbf{x} \mapsto \phi_{1}(\mathbf{x})=\left[\begin{array}{c}
\mathbf{x}_{1}+1 \\
\mathbf{x}_{2}+1
\end{array}\right] \\
& \mathbf{x} \mapsto \phi_{2}(\mathbf{x})=\left[\begin{array}{c}
\mathbf{x}_{1}-1 \\
\mathbf{x}_{2}
\end{array}\right]
\end{aligned}
$$

with corresponding transition intensities given by $\lambda_{1}(\mathbf{x})=$ $c_{1} \mathbf{x}_{1}$ and $\lambda_{2}(\mathbf{x})=c_{2} \mathbf{x}_{1} \mathbf{x}_{2}$ respectively. We are interested in estimating the time evolution of the means $\mathbf{E}\left[\mathbf{x}_{1}\right], \mathbf{E}\left[\mathbf{x}_{2}\right]$ and the following cumulants up to order three:

$$
\begin{aligned}
& \kappa^{(2,0)}=\mu^{(2,0)}-\mu^{(1,0)^{2}} \\
& \kappa^{(0,2)}=\mu^{(0,2)}-\mu^{(0,1)^{2}} \\
& \kappa^{(1,1)}=\mu^{(1,1)}-\mu^{(1,0)} \mu^{(0,1)} \\
& \kappa^{(3,0)}=2 \mu^{(1,0)^{3}}-3 \mu^{(1,0)} \mu^{(2,0)}+\mu^{(3,0)} \\
& \kappa^{(0,3)}=2 \mu^{(0,1)^{3}}-3 \mu^{(0,1)} \mu^{(0,2)}+\mu^{(0,3)} \\
& \kappa^{(2,1)}=2 \mu^{(0,1)} \mu^{(1,0)^{2}}-2 \mu^{(1,0)} \mu^{(1,1)}-\mu^{(0,1)} \mu^{(2,0)} \\
& \kappa^{(1,2)}=2 \mu^{(1,0)} \mu^{(0,1)^{2}}-2 \mu^{(0,1)} \mu^{(1,1)}-\mu^{(1,0)} \mu^{(0,2)} \mu^{(1,2)}
\end{aligned}
$$

We consider a third order truncation $(\mathscr{M}=3)$ with vector $\mu$ defined as in (28). From (14) the evolution of $\mu$ is given by

$$
\dot{\mu}=A \mu+B \bar{\mu}
$$

for some matrices $A$ and $B$ with

$$
\bar{\mu}=\left[\mu^{(3,1)}, \mu^{(1,3)}, \mu^{(2,2)}\right]^{T} .
$$

Using Table III, we obtain the following the truncated moment dynamics:

$$
\dot{v}=A v+B \bar{\varphi}(v), \bar{\varphi}(v)=\left[\begin{array}{c}
\left(\frac{v^{(2,1)}}{v^{(1,1)}}\right)^{3}\left(\frac{v^{(1,0)}}{v^{(2,0)}}\right)^{3} v^{(3,0)} v^{(0,1)} \\
\left(\frac{v^{(1,2)}}{v^{(1,1)}}\right)^{3}\left(\frac{v^{(0,1)}}{v^{(0,2)}}\right)^{3} v^{(0,3)} v^{(1,0)} \\
\frac{\left(v^{(2,1)} v^{(1,2)} v^{(0,1)} v^{(1,0)}\right)^{2}}{\left(v^{(1,1)}\right)^{4} v^{(2,0)} v^{(0,2)}}
\end{array}\right] .
$$


where

$$
\begin{aligned}
& \boldsymbol{v}=\left[\boldsymbol{v}^{(1,0)}, \boldsymbol{v}^{(0,1)}, \boldsymbol{v}^{(2,0)}, \boldsymbol{v}^{(0,2)}, \boldsymbol{v}^{(1,1)}, \boldsymbol{v}^{(3,0)}, \boldsymbol{v}^{(0,3)}, \boldsymbol{v}^{(2,1)}\right. \\
& \left.v^{(1,2)}\right]^{T}
\end{aligned}
$$

with the $p^{t h}$ element of $v(t)$ being an estimate for the $p^{t h}$ element of vector $\mu(t)$. Approximate cumulant time evolutions are then obtained by substituting the appropriate element of $v$ in (29). These are compared with estimates of cumulants obtained from averaging a large number of Monte Carlo simulations. All Monte Carlo simulations were carried out using the algorithm described in [6], which is equivalent to Gillespie's Monte Carlo simulation (SSA) procedure for chemical reactions [4]. Figures 1 shows cumulant $\kappa^{(0,3)}(t)$ obtained from Monte Carlo simulations and the truncated model (30) for initial conditions

$$
\mathbf{x}_{1}(0)=20, \quad \mathbf{x}_{2}(0)=10
$$

and $c_{1}=c_{2}=1$. For comparison purpose cumulant $\kappa^{(0,3)}(t)$ obtained from a second order of truncation $(\mathscr{M}=2)$ is also shown. The above truncated moment dynamics provides extremely good estimates for the time evolution of all cumulants of $\mathbf{x}$ up to order three. Due to lack of space constraints, estimates of only $\kappa^{(0,3)}(t)$ are provided.

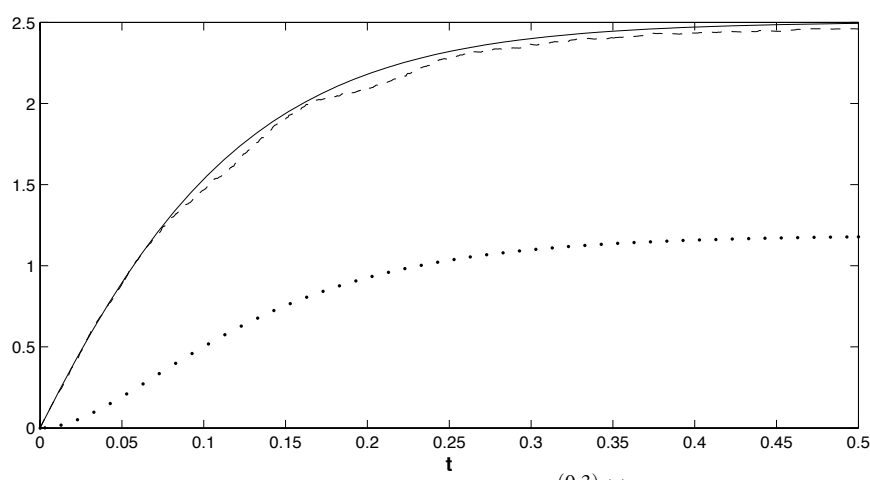

Fig. 1. Time evolution of the cumulant $\kappa^{(0,3)}(t)$ from Monte Carlo simulations (dashed line), from a third order truncation (30) (solid line) and from a second order truncation (dotted line).

\section{CONCLusion AND Future Work}

A procedure for constructing moment closures for chemically reacting systems was presented. This was done by first assuming a separable form for the moment closure function for each element of $\bar{\mu}$, and then, matching its time derivatives with $\bar{\mu}$, at some initial time $t_{0}$ for a basis of initial conditions. Explicit formulas to construct these moment closure functions for arbitrary order of truncation $\mathscr{M}$ were provided. The moment closure functions obtained are independent of the reaction parameters and the dependence of higher-order moment on lower order ones is consistent with the population being jointly lognormally distributed. In the last section we considered a simple set of bi-molecular reactions. The truncated moment dynamics provided fairly good estimates for the time evolution of all the cumulants of $\mathbf{x}$ up to order three when compared with estimates of cumulants obtained from averaging a large number of Monte Carlo simulations.

It was shown in [10] that for single specie reactions, there exists moments closure functions for which we actually have $\varepsilon_{2}(\overline{\mathbf{x}})=0$ in (27). A possible direction of future work would be to investigate the existence of such moment closure functions for multi-species reactions. Another direction of future work is to use the moment closure techniques discussed in this paper to study problems in population biology, such as, stochastic epidemics [13] and problems in system biology such as the bacteriophage $\lambda$ switch [2].

\section{REFERENCES}

[1] H. H. McAdams and A. P. Arkin, "Stochastic mechanisms in gene expression," Proceedings of the National Academy of Sciences U.S.A, vol. 94, pp. 814-819, 1997.

[2] J. Hasty, J. Pradines, M. Dolnik, and J. J. Collins, "Noise-based switches and amplifiers for gene expression," Proceedings of the National Academy of Sciences U.S.A, vol. 97, pp. 2075-2080, 2000.

[3] N. G. V. Kampen, Stochastic Processes in Physics and Chemistry. Amsterdam, The Netherlands: Elsevier Science, 2001.

[4] D. T. Gillespie, "A general method for numerically simulating the stochastic time evolution of coupled chemical reactions," J. of Computational Physics, vol. 22, pp. 403-434, 1976.

[5] _ "Approximate accelerated stochastic simulation of chemically reacting systems," J. of Chemical Physics, vol. 115, no. 4, pp. 17161733, 2001

[6] J. P. Hespanha, "Stochastic hybrid systems: Applications to communication networks," in Hybrid Systems: Computation and Control, ser. Lect. Notes in Comput. Science, R. Alur and G. J. Pappas, Eds. Berlin: Springer-Verlag, Mar. 2004, no. 2993, pp. 387-401.

[7] J. P. Hespanha and A. Singh, "Stochastic models for chemically reacting systems using polynomial stochastic hybrid systems," Int. J. of Robust and Nonlinear Control, vol. 15, pp. 669-689, 2005.

[8] A. Singh and J. P. Hespanha, "Models for multi-specie chemical reactions using polynomial stochastic hybrid systems," in Proc. of the 44th Conf. on Decision and Control, Seville, Spain, 2005.

[9] F. Wilkinson, Chemical Kinetics and Reaction Mechanisms. New York: Van Nostrand Reinhold Co, 1980.

[10] A. Singh and J. P. Hespanha, "Moment closure for the stochastic logistic model," Center for Control, Dynamical Systems and Computation. University of California at Santa Barbara, Tech. Rep. CCEC-06-0117, 2006, available at http://www.uweb.ucsb.edu/ abhi/papers/tech/moment.pdf.

[11] - "Moment closure techniques for stochastic models in population biology," in Proc. of the 2006 Amer. Control Conference, 2006.

[12] J. H. Matis, T. R. Kiffe, T. I. Matis, and D. E. Stevenson, "Nonlinear stochastic modeling of aphid population growth," Mathematical Bioscience, vol. 198, pp. 148-168, 2005.

[13] I. Krishnarajah, A. Cook, G. Marion, and G. Gibson, "Novel moment closure approximations in stochastic epidemics," Bulletin of Mathematical Biology, vol. 67, pp. 855-873, 2005. 